\title{
Cariótipo Fetal em Líquido Pleural Obtido por Toracocentese
}

\author{
Fetal Karyotyping of Pleural Fluid Obtained by Thoracocentesis \\ Antonio Carlos Vieira Cabral, Isabela Nelly Machado, Henrique Vitor Leite \\ Alamanda Kfoury Pereira, Zilma Nogueira Reis Vitral
}

\begin{abstract}
RESUMO
Objetivos: avaliar a efetividade da realização de cariótipo em liquido pleural obtido por toracocentese de um grupo de fetos portadores de derrame pleural.

Métodos: foram avaliados 15 fetos com derrame pleural uni ou bilateral nos quais se realizou uma punção da cavidade torácica. A idade gestacional variou entre 19 e 34 semanas. Os fetos foram estudados com ultra-sonografia morfológica para determinar a presença de anomalias associadas. Nos casos em que não se obteve o cultivo em líquido pleural foi realizada cordocentese para a realização do cariótipo. O líquido pleural obtido foi enviado para cultura de linfócitos e cariótipo, sendo a técnica de cultivo semelhante à realizada com sangue.

Resultados: dos 15 casos estudados foi obtido cariótipo em 12, e destes, em quatro se encontrou a trissomia do cromossomo 21 e os outros 8 foram normais. Em todos os casos, normais ou alterados, o cariótipo foi confirmado no sangue dos recém-nascidos e por meio do exame neonatal ou da necropsia. Não ocorreram complicações maternas ou fetais relacionadas ao procedimento invasivo.

Conclusões: o cariótipo em líquido pleural obtido por toracocentese mostrou ser procedimento eficaz e seguro, devendo ser empregado nos casos de fetos com derrame pleural.
\end{abstract}

PALAVRAS-CHAVE: Cariótipo. Toracocentese fetal. Anomalia cromossômica. Malformações fetais. Diagnóstico pré-natal.

\section{Introdução}

O acúmulo de líquido na cavidade pleural do feto é uma alteração geralmente benigna mas que em aproximadamente $50 \%$ dos casos pode ser causa de óbito fetal ou neonatal, relacionados principalmente aos quadros de hidropisia fetal nãoimunitária, parto prematuro e hipoplasia pulmonar. O que muda em relação ao prognóstico perinatal é o fator etiológico e não a resposta ao tratamento realizado, seja no período pré ou pósnatal $^{1}$.

Várias são as causas relacionadas ao derrame pleural fetal, sendo citadas como as mais freqüentes: anomalias estruturais (anomalias cardiacas), anormalidades cromossômicas (trissomia do cromossomo 21 e monossomia X), doenças infecciosas (adenovirus tipo 3, citomegalovírus) e

Centro de Medicina Fetal do Hospital das Clínicas da Universidade Federal de Minas Gerais

Correspondência: Antonio Carlos Vieira Cabral

Rua dos Otoni 909, sala 1804

30150-270 - Belo Horizonte - MG outras (fístula traqueoesofágica, síndrome de Noonan, etc.) $)^{1}$. Os protocolos que se propõem a avaliar fetos com derrame pleural devem sempre levar em consideração a possibilidade de doenças cromossômicas. Portanto, diante de fetos portadores de derrame pleural isolado ou como parte do quadro de hidropisia não-imunitária o cariótipo se faz de grande valor para avaliar a possibilidade terapêutica e o prognóstico, além do risco de recorrência ${ }^{1-4}$.

São complicações associadas ao derrame pleural o polidrâmnio e a hipoplasia pulmonar. $\mathrm{O}$ polidrâmnio ocorre seja devido a uma redução da deglutição fetal ou mesmo pela insuficiência cardíaca secundária à compressão dos vasos da base nos casos em que o mediastino se encontra desviado. É comum nos casos de compressão dos vasos da base o desenvolvimento da hidropisia fetal não-imunitária. A hipoplasia pulmonar ocorre pela compressão extrínseca do parênquima pulmonar, que impede o seu desenvolvimento normal. Portanto, a abordagem do derrame pleural também visa não apenas a identificação do fator causal 
como também, quando possível, prevenir a hipoplasia pulmonar, a hidropisia fetal e suas conseqüências ${ }^{1,4}$.

A análise citogenética antenatal é tradicionalmente realizada em células obtidas do vilo corial, líquido amniótico e sangue fetal. Recentemente alguns estudos cromossômicos passaram a ser realizados em células fetais obtidas em locais não-convencionais, tais como líquido pleural, ascítico, urina e líquido de higroma cístico ${ }^{2}$.

No presente trabalho apresentamos a experiência do Centro de Medicina Fetal do Hospital das Clínicas com a realização do cariótipo em líquido de derrame pleural.

\section{Pacientes e Métodos}

Trata-se de um estudo prospectivo em que se avaliou de janeiro de 1997 a dezembro de 1999 um grupo de 15 fetos com derrame pleural uni ou bilateral acompanhados no Centro de Medicina Fetal do Hospital das Clínicas da Universidade Federal de Minas Gerais. Nestes fetos foi tentada a realização do cariótipo no líquido pleural obtido por meio de toracocentese. O trabalho foi previamente aprovado na Comissão de Ética do Serviço e faz parte do protocolo de acompanhamento de fetos com hidropisia fetal não-imunitária ou derrame pleural isolado.

A punção torácica nos casos de fetos com derrame pleural foi realizada como propedêutica (pesquisa genética e infecciosa) e terapêutica, já que em alguns casos não ocorre o reacúmulo após a punção ${ }^{5}$.

Inicialmente foi explicado o procedimento, sua importância e complicações possiveis e, após consentimento, as pacientes foram submetidas ao procedimento invasivo guiado por ultra-som.

A punção torácica foi realizada sob visão ultra-sonográfica com a utilização de agulha BD 20 gauge com $15 \mathrm{~cm}$ de comprimento. Em alguns casos de polidrâmnio muito volumoso utilizou-se uma agulha BD 20 gauge com $20 \mathrm{~cm}$ de comprimento. Após anti-sepsia com Povidine, anestesia com Xylocaína a $2 \%$, sem vasoconstritor, com a orientação do ultra-som foi realizada a punção do tórax fetal e aspirado o líquido pleural. Este foi enviado em seringa descartável para realização de citometria (esfregaço em lâmina). Quando o número de linfócitos foi superior a $60 \%$ o material foi encaminhado para cultivo e cariótipo por técnica convencional ${ }^{3}$. Nos casos em que o número de linfócitos foi inferior a $60 \%$ foi realizada a cordocentese e o sangue encaminhado para cariotipagem.
A idade gestacional no momento do procedimento variou de 19 a 34 semanas, sendo que 6 gestações tinham menos de 30 semanas e as demais acima desta idade gestacional.

O volume obtido variou de 10 a $103 \mathrm{~mL}$, com valor médio de $20 \mathrm{~mL}$ por punção, sendo que sempre se objetivava o total esvaziamento do tórax.

\section{Resultados}

Na Tabela 1 são apresentados os casos estudados. Podemos observar que dos 15 casos, em 3 não se obteve o cultivo de forma satisfatória. Dos casos em que se obteve o cariótipo, em 4 o exame se apresentou alterado. Em todos estes 4 estava presente a trissomia do cromossomo 21 e nos outros 8 o cariótipo se revelou normal.

Tabela 1 - Características e resultados citogenéticos de quinze fetos submetidos a toracocentese para cultivo do líquido pleural.

\begin{tabular}{rlll}
\hline Caso & IG* $^{*}$ & Cultivo & \multicolumn{1}{c}{ Cariótipo } \\
\hline 1 & 28 & Positivo & $46 \mathrm{XY}$ \\
2 & 30 & Positivo & $47 \mathrm{XY}+21$ \\
3 & 33 & Positivo & $46 \mathrm{XX}$ \\
4 & 28 & Positivo & $47 \mathrm{XX}+21$ \\
5 & 33 & Positivo & $46 \mathrm{XX}$ \\
6 & 31 & Negativo & - \\
7 & 34 & Positivo & $46 \mathrm{XY}$ \\
8 & 33 & Negativo & - \\
9 & 29 & Positivo & $46 \mathrm{XY}$ \\
10 & 19 & Positivo & $47 X X+21$ \\
11 & 25 & Negativo & - \\
12 & 33 & Positivo & $46 \mathrm{XX}$ \\
13 & 30 & Positivo & $47 X Y+21$ \\
14 & 27 & Positivo & $46 \mathrm{XY}$ \\
15 & 33 & Positivo & $46 \mathrm{XY}$ \\
\hline
\end{tabular}

IG - Idade gestacional da punção torácica fetal.

Todos os neonatos vivos foram submetidos a novo cariótipo em sangue obtido após o parto para confirmação dos achados obtidos no líquido amniótico (7/ 15 casos). Os demais fetos, 8 casos, foram submetidos a necropsia, que revelou no caso 2 a presença de comunicação interventricular (CIV) e atresia duodenal e no caso 10 asplenia e edema nucal. Observamos no caso 14 um achado na placenta compativel com doença do metabolismo por elevada concentração citoplasmática de substância amorfa. Ao realizarmos estudo nos pais, 
chegou-se ao diagnóstico de gangliosidose tipo GM 1.

Nos 3 casos em que não se obteve crescimento celular, o óbito fetal ocorreu entre 2 e 3 semanas após a punção. O caso 6 revelou, à necropsia, tratar-se de doença adenomatosa cística pulmonar, com área de seqüestro lobar. No caso 11 nenhuma anomalia fetal foi identificada além do derrame pleural, permanecendo sem diagnóstico etiológico. No caso 8 a família não permitiu a realização da necropsia, sendo que no exame ultra-sonográfico e no ecocardiograma prénatal haviam sido encontrados, além do derrame pleural, o encurtamento dos ossos longos (fêmur) e uma comunicação interventricular, além da nãovisualização das válvulas átrio-ventriculares.

Nenhuma complicação obstétrica nos casos acompanhados foi relacionada à toracocentese. Verificamos no caso 4 parto prematuro seis semanas após a realização da punção, sendo o desencadeamento imputado ao polidrâmnio acentuado.

\section{Discussão}

A realização da toracocentese fetal para esvaziamento pleural tem sido proposta há vários anos com finalidade terapêtica ${ }^{5}$. Sabe-se que o esvaziamento do tórax fetal é medida preventiva da hipoplasia pulmonar, promovendo redução do líquido amniótico nos casos de polidrâmnio e evitando complicações da parturição ${ }^{4-6}$. A redução do polidrâmnio esta relacionada à normalização da posição do mediastino e facilitação da deglutição do líquido amniótico pelo feto.

A utilização do líquido pleural para análise citogenética tem sido realizada em adultos há muitos anos com pleno sucesso ${ }^{7}$, sendo que passou também a ser feita em casos de fetos com a mesma eficácia ${ }^{2,3}$.

Uma das maiores vantagens desta fonte de estudo citogenético está na baixa contaminação com células maternas, além de o tempo de realização da cariotipagem ser rápido, podendo o resultado ser obtido em 2 a 3 dias $^{2}$.

Nossos resultados mostraram um elevado indice de cultivo celular em líquido pleural, com o sucesso na obtenção do cultivo em $80 \%$ dos casos. Evidenciamos uma segurança muito elevada do resultado, semelhante ao verificado em outros estudos em que todos os resultados (alterados ou normais) foram confirmados na avaliação pós-natal ${ }^{2}$.

Dos 15 casos com derrame pleural, a trissomia do cromossomo 21 esteve presente em $26,7 \%$ (4 fetos), mostrando a associação freqüente desta aneuploidia com o derrame pleural. Nicolaides e Azar ${ }^{8}$ encontraram uma incidência em torno de $11 \%$ de trissomia do cromossomo 21 em fetos com derrame pleural.

Acreditamos que diante de fetos com derrame pleural, a opção pela toracocentese deve ser primeiramente considerada. Permite estudo citogenético com segurança semelhante à obtida em métodos convencionais, como a cordocentese, além de apresentar possibilidades de ações terapêuticas como a prevenção da hipoplasia pulmonar.

Concluímos que o cariótipo em líquido pleural obtido por toracocentese mostrou ser procedimento de grande eficácia e segurança, devendo ser realizado nos casos de fetos com derrame pleural uni ou bilateral.

\section{SUMMARY}

Purpose: to evaluate the possibility and accuracy of fetal karyotyping in pleural effusions.

Methods: we studied fifteen fetuses with unilateral or bilateral pleural effusions. All of these fetuses underwent intrauterine thoracocentesis guided by ultrasound examinations. The gestational age varied from 19 to 34 weeks. A morphogenetic ultrasound examination was performed in each case by the authors in order to identify associated structural anomalies. When the cellular cultures of pleural effusion samples were negative, an alternative karyotype was obtained by cordocentesis. A fetal lymphocyte culture was made of pleural effusion samples for karyotype in a similar technique as for fetal blood.

Results: the fetal karyotype was successful in 12 cases. There were 4 abnormal results, all of them were Down syndromes, and in the other 8 cases the chromosomal analyses were normal. The fetal karyotype was confirmed and compared by newborn blood chromosomal analysis, genetic evaluation or necropsy. There were no maternal or fetal side effects related to the procedure.

Conclusions: the fetal karyotyping performed in pleural effusions obtained by intrauterine thoracocentesis proved to be highly efficient and safe. It must be the method of choice for rapid karyotyping in fetuses with pleural edema.

KEY WORDS: Karyotype. Fetal thoracocentesis. Chromosomal abnormalities. Fetal structural anomalies. Prenatal diagnosis. 


\section{Referências}

1. Vaughan JI, Fisk NM, Rodeck CH. Fetal pleural effusions. In: Harman CR, editor. Invasive Fetal Testing and Treatment. $1^{\text {st }}$ ed. Boston: Blackwell Scientific; 1995. p.219-39.

2. Teoh TG, Ryan G, Johnson J, Winsor EJ. The role of fetal karyotyping from unconventional sources. Am J Obstet Gynecol 1996; 175:873-7.

3. Cabral ACV, Leite HV, Corbani M, Pereira AK. Toracocentese fetal para cariótipo em líquido pleural. J Bras Ginecol 1994; 104:429-30.

4. Lange IR, Manning FA. Antenatal diagnosis of congenital pleural effusions. Am J Obstet Gynecol 1981; 140:839-40.
5. Cabral ACV, Leite HV, Aguiar RALP. Toracocentese fetal no tratamento da efusão pleural: relato de um caso. J Bras Ginecol 1992; 102:299-300.

6. Rodeck CH, Fisk NM, Fraser DI, Nicolini U. Longterm in utero drainage of fetal hydrotorax. N Engl J Med 1988; 319:1135-8.

7. Giazza G, Cosimi MF, Lanero M, Rossi GP, Casagranda I. Cytologic diagnosis and chromosome analysis: sensitivity, specificity, accuracy and predictive value in malignant and benign pleural effusions. Pathologica 1990; 82:33-40.

8. Nicolaides KH, Azar GB. Thoraco-amniotic shunting. Fetal Diagn Ther 1990; 5:153-64.

\section{COMUNICADO AOS ASSOCIADOS E LEITORES}

\section{Para informações e consultas temos à disposição quatro endereços eletrônicos:}

\section{febrasgopresiden@uol.com.br} secretaria_executiva@febrasgo.org.br publicacoes@febrasgo.org.br tego_habilitacao@febrasgo.org.br 